\title{
Switching between bistable states in a discrete nonlinear model with long-range dispersion
}

Johansson, Magnus; Gaididei, Yuri B.; Christiansen, Peter Leth; Rasmussen, Kim

Published in:

Physical Review E. Statistical, Nonlinear, and Soft Matter Physics

Link to article, DOI:

10.1103/PhysRevE.57.4739

Publication date:

1998

Document Version

Publisher's PDF, also known as Version of record

Link back to DTU Orbit

Citation (APA):

Johansson, M., Gaididei, Y. B., Christiansen, P. L., \& Rasmussen, K. (1998). Switching between bistable states in a discrete nonlinear model with long-range dispersion. Physical Review E. Statistical, Nonlinear, and Soft Matter Physics, 57(4), 4739-4742. https://doi.org/10.1103/PhysRevE.57.4739

\section{General rights}

Copyright and moral rights for the publications made accessible in the public portal are retained by the authors and/or other copyright owners and it is a condition of accessing publications that users recognise and abide by the legal requirements associated with these rights.

- Users may download and print one copy of any publication from the public portal for the purpose of private study or research.

- You may not further distribute the material or use it for any profit-making activity or commercial gain

- You may freely distribute the URL identifying the publication in the public portal 


\title{
Switching between bistable states in a discrete nonlinear model with long-range dispersion
}

\author{
Magnus Johansson, ${ }^{1}$ Yuri B. Gaididei, ${ }^{2}$ Peter L. Christiansen, ${ }^{1}$ and K. $\emptyset$. Rasmussen ${ }^{1}$ \\ ${ }^{1}$ Department of Mathematical Modelling, The Technical University of Denmark, DK-2800 Lyngby, Denmark \\ ${ }^{2}$ Institute for Theoretical Physics, Metrologicheskaya Street 14B, 252143 Kiev 143, Ukraine
}

(Received 26 September 1997; revised manuscript received 8 December 1997)

\begin{abstract}
In the framework of a discrete nonlinear Schrödinger equation with long-range dispersion, we propose a general mechanism for obtaining a controlled switching between bistable localized excitations. We show that the application of a spatially symmetric kick leads to the excitation of an internal breathing mode and that switching between narrow, pinned states and broad, mobile states with only small radiative losses occurs when the kick strength exceeds a threshold value. This mechanism could be important for controlling energy storage and transport in molecular systems. [S1063-651X(98)01004-6]

PACS number(s): 63.20.Pw, 63.20.Ry
\end{abstract}

Describing the storage and transport of energy and charge in the presence of discreteness, dispersion, and selfinteraction is a problem of major importance in solid-state physics, biophysics, and optics. Applications include, e.g., polaron formation in electron-lattice coupled systems, localization of vibrational energy in proteins, and localization of optical beams in arrays of nonlinear waveguides. The discrete nonlinear Schrödinger (DNLS) equation has been used extensively in the literature to model these phenomena and it is known to have exact spatially localized, time-periodic solutions (breathers) that are stationary states [1-4]. However, since the standard DNLS equation only includes dispersive coupling between nearest neighbors, it does not appropriately describe situations where long-range interactions are important (e.g., the DNA molecule contains charged groups and therefore the vibration-excitation transfer is due to dipole-dipole interactions decaying with distance $r$ as $1 / r^{3}$ ). To describe such situations, a DNLS equation with longrange coupling was proposed in [5]. With the dispersive coupling decaying as $1 / r^{s}$ the coexistence of three on-site localized stationary states having the same value of the norm was found when $2<s \lesssim 3.03$. A further analysis showed that in the interval of multistability, the three different states could be classified as one stable, broad ("continuumlike") state with high mobility; one unstable, intermediate state; and one stable, narrow ("intrinsically localized" or "discrete") state that is pinned to the lattice. This bistability phenomenon is generic (e.g., for exponentially decreasing coupling $e^{-\beta r}$ it occurs when $\beta \leq 1.70$ ) and can be understood as the result of two competing length scales, one due to the long-range nature of the dispersive coupling and the other resulting from the balance between nonlinearity and dispersion.

Having established the existence of bistable stationary states in the nonlocal DNLS system, a natural question that arises concerns the possibility of switching between the stable states under the influence of some external perturbations. Switching of this type is important in the description of nonlinear transport and storage of energy in biomolecules such as DNA since a mobile continuumlike state can provide action at a distance, while the switching to a discrete, pinned state can facilitate the structural changes of DNA [6]. It is the purpose of the present paper to show that switching will occur if the system is perturbed in a way so that an internal, spatially localized and symmetric mode ("breathing mode") of the stationary state is excited above a threshold value. The importance of internal modes for the dynamics of nonlinear localized excitations in both discrete [7,8] and continuum [9] systems has been emphasized recently. In particular, it was shown in Ref. [7] that a pinned, discrete breather could become mobile by exciting a spatially antisymmetric translational ("pinning") mode above a threshold value. Thus we find that the role of the breathing mode in the switching process is analogous to the role of the translational mode in the depinning process.

We consider the nonlocal discrete nonlinear Schrödinger equation of the form

$$
i \dot{\psi}_{n}+\sum_{m \neq n} J_{n-m}\left(\psi_{m}-\psi_{n}\right)+\left|\psi_{n}\right|^{2} \psi_{n}=0
$$

where the long-range dispersive coupling is taken to be either exponentially $J_{n}=J e^{-\beta|n|}$ or algebraically $J_{n}=J|n|^{-s}$ decreasing with distance $n \neq 0$. In both cases, the constant $J$ will be normalized such that $\sum_{n=1}^{\infty} J_{n}=1$ for all $\beta$ or $s$. The ordinary nearest-neighbor DNLS equation is then recovered in the limits $\beta \rightarrow \infty$ and $s \rightarrow \infty$, respectively. Like the ordinary DNLS equation, Eq. (1) has two conserved quantities, namely, the norm (excitation number) $\mathcal{N}=\Sigma_{n}\left|\psi_{n}\right|^{2}$ and the Hamiltonian [5].

Stationary states $\psi_{n}^{(\Lambda)}(t)$ are time-periodic solutions to Eq. (1) of the form $\psi_{n}^{(\Lambda)}(t)=\phi_{n} e^{i \Lambda t}$, where $\phi_{n}$ is time independent. To investigate the time evolution of an initially small perturbation $\varepsilon_{n}(0)$ of a stationary state, we follow the approach in [1] and write $\psi_{n}(t)=e^{i \Lambda t}\left[\phi_{n}+\varepsilon_{n}(t)\right]$. Decomposing $\varepsilon_{n}$ into real and imaginary parts $\varepsilon_{n}^{(r)}$ and $\varepsilon_{n}^{(i)}$ and linearizing around $\psi_{n}^{(\Lambda)}(t)$, we obtain

$$
\frac{d}{d t}\left(\begin{array}{c}
\varepsilon_{n}^{(r)} \\
\varepsilon_{n}^{(i)}
\end{array}\right)=\mathcal{M}\left(\begin{array}{c}
\varepsilon_{n}^{(r)} \\
\varepsilon_{n}^{(i)}
\end{array}\right)=\left(\begin{array}{cc}
0 & H^{+} \\
-H^{-} & 0
\end{array}\right)\left(\begin{array}{l}
\varepsilon_{n}^{(r)} \\
\varepsilon_{n}^{(i)}
\end{array}\right),
$$

where for a system with $N$ sites, $H^{+}$and $H^{-}$are $N \times N$ matrices defined by $H_{i j}^{ \pm}=\left[\Lambda-(2 \mp 1) \phi_{i}^{2}+2\right] \delta_{i, j}-J_{i-j}$ (with $J_{0}=0$ ). Thus we obtain information about the dynamics close to the stationary state by studying the eigenvalues $\mu$ and eigenvectors $\left(\xi_{n}, \eta_{n}\right)^{T}$ of the $2 N \times 2 N$ matrix $\mathcal{M}[1,10]$. 
By definition, the solution $\psi_{n}^{(\Lambda)}(t)$ is linearly stable if the perturbation $\varepsilon_{n}(t)$ as calculated from Eq. (2) remains bounded for all times. Linear stability is then equivalent to the matrix $\mathcal{M}$ having no eigenvalues with a positive real part, which, due to the symplectic nature of Eq. (2), means that all its eigenvalues must be located as complex-conjugate pairs on the imaginary axis. By changing some parameter (e.g., $\Lambda$ or $J$ ), a stable state might become unstable, which is seen as two eigenvalues of the matrix $\mathcal{M}$ colliding and leaving the imaginary axis. The "direction" in which an initial perturbation will grow is then determined by the eigenvector corresponding to the eigenvalue of $\mathcal{M}$ with a positive real part. In the stable case, the eigenvectors corresponding to eigenvalues of $\mathcal{M}$ on the imaginary axis can be of two different types: extended, corresponding to a continuous spectrum in the limit $N \rightarrow \infty$, or localized, corresponding to a discrete spectrum. We will here be particularly interested in the localized eigenvectors since they correspond to the internal modes of the stationary states through which the switching between states can occur. Since the location of the continuous spectrum $\mu(k)$ can be analytically determined from the linear dispersion relation of Eq. (2) with $\phi_{n} \equiv 0$, we can numerically identify the localized eigenmodes as corresponding to eigenvalues outside the linear band. For example, when the coupling is exponentially decreasing with exponent $\beta$, the continuous spectrum is given by the intervals on the imaginary axis where $|\mu(k)| \in[\Lambda, \Lambda+4(1$ $\left.\left.+e^{-\beta}\right)^{-1}\right]$.

As was shown in Ref. [5], stationary states that are spatially symmetric and localized with a single maximum at a lattice site ("on-site" states or "single-site breathers") are linearly stable if and only if the well-known $[3,12]$ condition $\partial \mathcal{N} / \partial \Lambda>0$ is fulfilled. In Fig. 1(a) we show the dependence $\mathcal{N}(\Lambda)$ for the on-site state in the case of exponentially decreasing coupling $J_{n}$ (the corresponding curve for algebraically decreasing coupling was plotted as Fig. 2 in Ref. [5]). For $\beta \gtrsim 1.70$ the norm is a monotonically increasing function of the frequency $\Lambda$, implying that the state is linearly stable for all $\Lambda$, while for $\beta \leqq 1.70$ the curve is nonmonotonic, implying the simultaneous existence of three stationary states with different frequencies, two of which are stable and one unstable, having the same norm for some values of $\mathcal{N}$. To be specific, we will in the following mainly discuss the case $\beta$ $=1.0$, where multistability occurs in the interval $3.23 \leq \mathcal{N}$ $\$ 3.78$.

The results obtained from a numerical diagonalization of the matrix $\mathcal{M}$ in Eq. (2) for $\beta=1.0$ are illustrated in Fig. 1(b). We find that for large values of $\Lambda(\Lambda \gtrsim 1.93)$, i.e., when the stationary state is strongly localized, there is no discrete spectrum and consequently the stationary state has no localized internal mode. When $\Lambda \approx 1.93$, an eigenvalue $\mu^{(s)}$ corresponding to a spatially symmetric breathing mode bifurcates off the band edge of the continuous spectrum at $k=0$. As is seen from Fig. 1(b), this eigenvalue approaches zero along the imaginary axis for a further decrease of $\Lambda$, and for $\Lambda \approx 0.76$ it becomes real and the state becomes unstable. Decreasing $\Lambda$ further results in an instability that grows until $\mu^{(s)}$ reaches a maximum value and then becomes weaker again as $\mu^{(s)}$ approaches zero. At $\Lambda \approx 0.39$ the eigenvalue returns to the imaginary axis and the state, which now has a
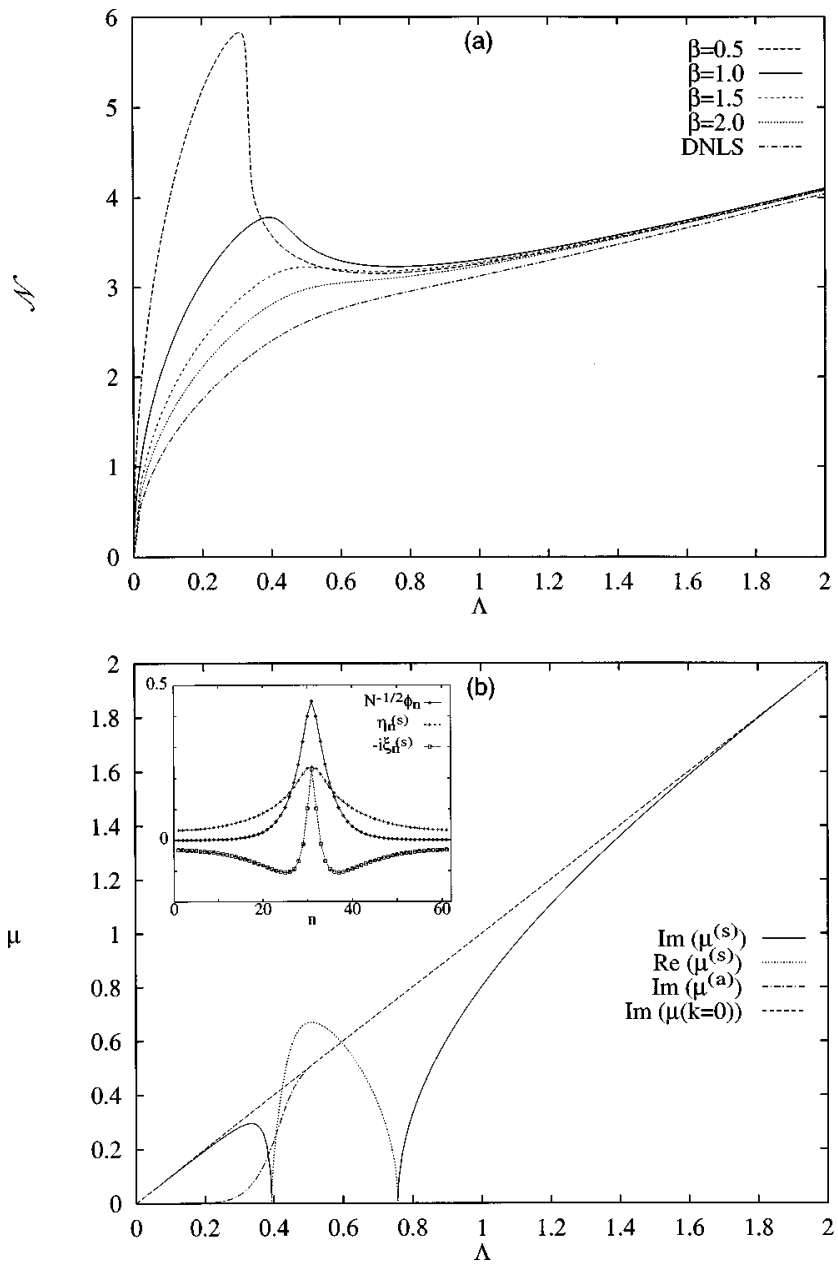

FIG. 1. (a) Dependence $\mathcal{N}(\Lambda)$ for stationary on-site localized solutions to Eq. (1) with $J_{n}$ exponentially decreasing. From top to bottom in the left part of the figure, $\beta=0.5, \beta=1.0, \beta=1.5, \beta$ $=2.0$, and $\beta \rightarrow \infty$, respectively. (b) Eigenvalues $\mu$ of the matrix $\mathcal{M}$ in Eq. (2) versus $\Lambda$ for $\beta=1.0$. The solid (dotted) line shows the imaginary (real) part of the eigenvalue $\mu^{(s)}$ corresponding to the spatially symmetric, localized mode; the dash-dotted line shows the imaginary part of the eigenvalue $\mu^{(a)}$ corresponding to the translational mode; the straight dashed line shows the lower bound of the continuous spectrum. (The spectrum is symmetric around $\mu=0$.) The inset in (b) shows, from top to bottom at $n=n_{0}=31, \phi_{n} / \sqrt{\mathcal{N}}$, $\eta_{n}^{(s)}$, and $-i \xi_{n}^{(s)}$, respectively, where $\phi_{n}$ is the stationary state used as the initial condition in Fig. 2(a) and $\left(\xi_{n}^{(s)}, \eta_{n}^{(s)}\right)^{T}$ is the corresponding normalized eigenvector of the matrix $\mathcal{M}$ with eigenvalue $\mu^{(s)} \approx 0.286 i$ (choosing the overall phase so that $\eta_{n}^{(s)}$ is real and positive).

continuumlike nature, is again stable. As $\Lambda \rightarrow 0, \mu^{(s)}$ approaches the band edge of the continuous spectrum, but we find that a localized breathing mode exists also close to the continuum limit. Furthermore, as is seen in Fig. 1(b), a second eigenvalue $\mu^{(a)}$, corresponding to a spatially antisymmetric translational mode, bifurcates from the $k=0$ band edge at $\Lambda \approx 0.51$. Since the appearance of a translational mode implies that the stationary state gains mobility that increases as $\mu^{(a)}$ approaches zero [7], the continuumlike state will have a high mobility (in particular for $\Lambda \leqq 0.25$ where $\mu^{(a)}$ is very close to zero).

The scenario described above and illustrated in Fig. 1(b) 

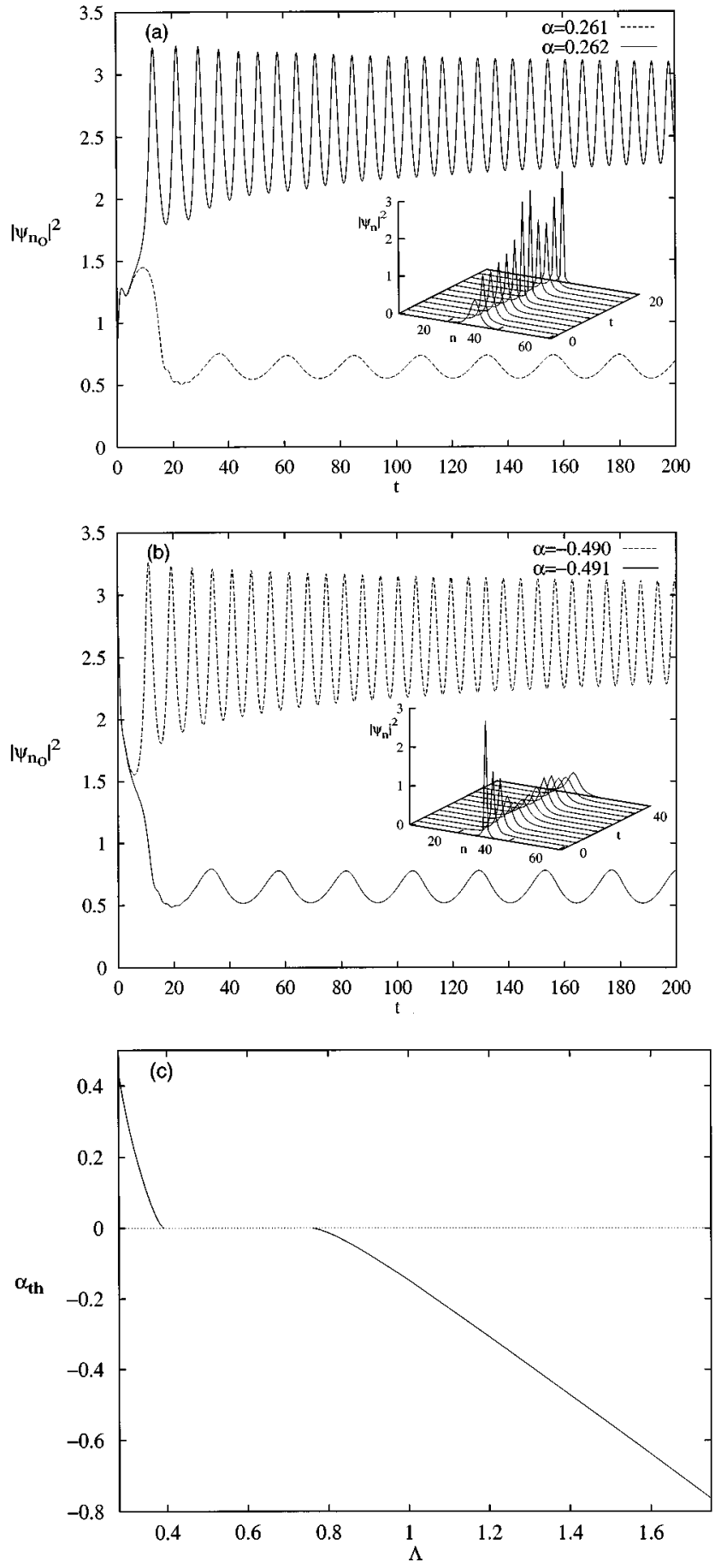

FIG. 2. (a) Switching from a continuumlike to a discrete state, where the initial state $\phi_{n}$ has frequency $\Lambda \approx 0.310$ and $\mathcal{N}=3.6$. The main figure shows the time evolution of $\left|\psi_{n_{0}}(t)\right|^{2}$ when a phase torsion is applied to the central site with $\alpha=0.261$ (lower curve) and $\alpha=0.262$ (upper curve), respectively; the inset shows the time evolution of $\left|\psi_{n}(t)\right|^{2}$ for $\alpha=0.262$. (b) Switching from a discrete to a continuumlike state, where the initial state $\phi_{n}$ has frequency $\Lambda$ $\approx 1.423$ and $\mathcal{N}=3$.6. The main figure shows the time evolution of $\left|\psi_{n_{0}}(t)\right|^{2}$ with $\alpha=-0.490$ (upper curve) and $\alpha=-0.491$ (lower curve), respectively; the inset shows the time evolution of $\left|\psi_{n}(t)\right|^{2}$ for $\alpha=-0.491$ (only a part of a larger system is shown). (c) Threshold value of the phase torsion $\alpha_{\text {th }}$ versus $\Lambda$. Switching occurs when $|\alpha|>\left|\alpha_{\mathrm{th}}\right|$ and $\alpha>0(\alpha<0)$ for an initial continuumlike (discrete) state. In all figures, $\beta=1.0$.

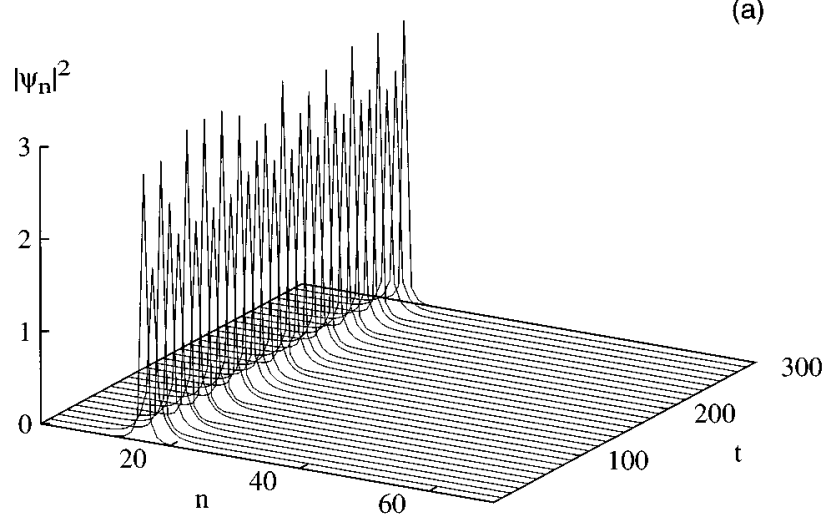

(b)

$\left|\psi_{n}\right|^{2}$

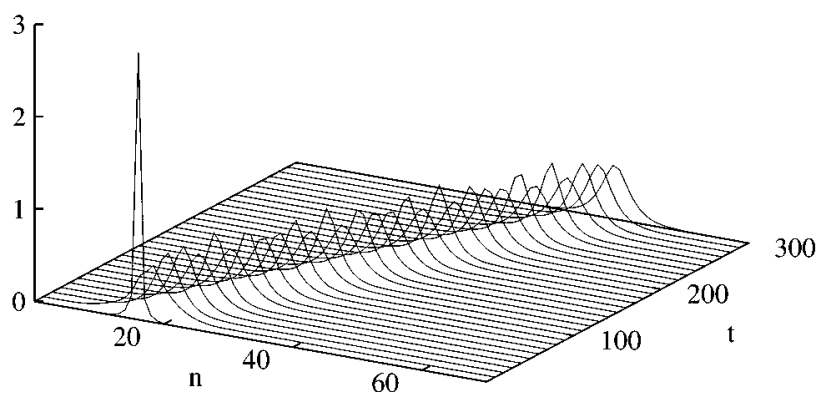

FIG. 3. Switching from a discrete state to a moving, continuumlike state. The initial state $\phi_{n}$ is the same as in Fig. 2(b), but with an initial perturbation containing also a spatially antisymmetric component $(\varkappa=-0.1)$. In (a), the phase torsion at the central site is just below threshold for switching ( $\alpha=-0.47$ ), while in (b) it is just above ( $\alpha=-0.48$ ) (only a small part of a larger system is shown). Note that the threshold for switching is slightly smaller than in Fig. 2(b).

remains qualitatively unchanged for all values of $\beta \leqslant 1.70$ and also for the algebraically decaying coupling with $2<s$ $\lesssim 3.03$, where multistability occurs. When increasing $\beta$ (or $s$ ) the maximum value attained by $\mu^{(s)}$ in the instability regime decreases; for $\beta \gtrsim 1.70(s \gtrsim 3.03) \mu^{(s)}$ stays imaginary for all $\Lambda$ where it exists and no instability develops.

An illustration of how the presence of an internal breathing mode affects the dynamics of a slightly perturbed stable stationary state is given in Fig. 2. To excite the breathing mode, we apply a spatially symmetric, localized perturbation, which we choose to be norm conserving in order not to change the effective nonlinearity of the system. The simplest choice, which we have used in the simulations shown here, is to kick the central site $n_{0}$ of the system at $t=0$ by adding a parametric force term of the form $\alpha \delta_{n, n_{0}} \delta(t) \psi_{n}$ to the lefthand side of Eq. (1). As can easily be shown, this perturbation affects only the site $n_{0}$ at $t=0$ and results in a "twist" of the stationary state at this site with an angle $\alpha$, i.e., $\psi_{n_{0}}(0)=\phi_{n_{0}} e^{i \alpha}$. From a biophysical point of view, such a kick may occur as a consequence of the interaction between a biomolecule and solvent molecules and ions (ligands). Namely, an instantaneous attachment and detachment of a ligand to the molecule leads to a local, instantaneous frequency change for its vibrational units, and in a rotatingwave approximation a DNLS equation with the additional 
parametric force term considered here can be derived [13].

The immediate consequence of the kick is, as can be deduced from the form of Eq. (2), that $(d / d t)\left(\left|\psi_{n_{0}}\right|^{2}\right)$ will be positive (negative) when $\alpha>0 \quad(\alpha<0)$. Thus, to obtain switching from the continuumlike state to the discrete state we choose $\alpha>0$, while we choose $\alpha<0$ when investigating switching in the opposite direction. We find that in a large part of the multistability regime there is a well-defined threshold value $\alpha_{\text {th }}$ such that when the initial phase torsion is smaller than $\alpha_{\text {th }}$ periodic, slowly decaying breather oscillations around the initial state will occur, while for strong enough kicks (phase torsions larger than $\alpha_{\mathrm{th}}$ ) the state switches into the other stable stationary state, around which breather oscillations develop [see Figs. 2(a) and 2(b)]. The numerically calculated dependence of $\alpha_{\text {th }}$ on $\Lambda$ is plotted in Fig. 2(c). Since also some extended eigenmodes are excited by the perturbation, there will be some radiation escaping to infinity as $t \rightarrow \infty$, so that the norm of the final state after the switching process will be slightly smaller than the initial norm. In the cases considered in Fig. 2, we estimate the norm of the final localized states to be approximately 3.5. Due to the radiative losses, the switching occurs only once when $\alpha$ is close to $\alpha_{\text {th }}$. However, when the phase torsion is considerably larger than the threshold value, we have also observed situations where multiple switching between the states occurs [14].

We stress that the particular choice of perturbation is not important for the qualitative features of the switching, as long as there is a substantial overlap between the perturbation and the internal breathing mode. For example, the breathing mode corresponding to the continuumlike state is spatially rather extended, as can be seen from the inset in Fig. 1(b), and thus an initial perturbation where the kick is applied to more than one site is a closer approximation to the mode. We found, e.g., that kicking the three central sites with the same strength $\alpha$ in this case gave a slightly lower threshold value $\alpha_{\text {th }}$, but no qualitative changes in the dynamics. The threshold value and the radiative losses would be minimized if the initial perturbation was chosen exactly in the direction of the breathing mode, but we consider such a perturbation to be somewhat artificial since in a real system such as a biomolecule the exact shape of the breathing mode in general cannot be assumed to be known. Also, we believe that the mechanism for switching described here can be applied for any multistable system where the instability is connected with a breathing mode. For example, we observed a similar switching behavior in the nearest-neighbor DNLS equation with a higher degree of nonlinearity, which is known [3] to exhibit multistability.

Since in the simulations discussed above the initial perturbations are spatially symmetric, also the potentially mobile broad states [e.g., the final state in Fig. 2(b)] remain static, their translational modes being unexcited. However, by including also a spatially nonsymmetric component in the perturbation, a moving, broad excitation will result if also the translational mode is excited above its threshold value [7]. (This could, e.g., be the result of including a small random noise corresponding to thermal fluctuations.) An example where the use of an initial perturbation having both a spatially symmetric and an antisymmetric part leads to a direct switching from a narrow, pinned state to a broad, moving state is shown in Fig. 3. Here the initial perturbation has the

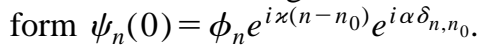

In conclusion, we have shown how the excitation of an internal breathing mode above a threshold value can lead to switching between bistable pinned and mobile nonlinear localized excitations and indicated how this mechanism may be used as a model for the control of storage and transport of energy in biomolecules.

We thank V. K. Mezentsev and J. Juul Rasmussen for discussions. Yu. B. G. thanks the Technical University of Denmark for hospitality and acknowledges support from the Ukrainian Fundamental Research Fund under Grant No. 2.4/ 355. M. J. acknowledges support from the Swedish Foundation STINT. The present work was supported by the Danish Research Council through Contract No. 9313393 (supercomputing projects).
[1] J. C. Eilbeck, P. S. Lomdahl, and A. C. Scott, Physica D 16, 318 (1985); J. Carr and J. C. Eilbeck, Phys. Lett. 109A, 201 (1985).

[2] R. S. MacKay and S. Aubry, Nonlinearity 7, 1623 (1994).

[3] E. W. Laedke, K. H. Spatschek, and S. K. Turitsyn, Phys. Rev. Lett. 73, 1055 (1994).

[4] S. Flach, K. Kladko, and R. S. MacKay, Phys. Rev. Lett. 78, 1207 (1997).

[5] Yu. B. Gaididei, S. F. Mingaleev, P. L. Christiansen, and K. Ф. Rasmussen, Phys. Rev. E 55, 6141 (1997).

[6] G. Gaeta, C. Reiss, M. Peyrard, and T. Dauxois, Riv. Nuovo Cimento 17, 1 (1994); S. Georghiou et al., Biophys. J. 70, 1909 (1996).

[7] Ding Chen, S. Aubry, and G. P. Tsironis, Phys. Rev. Lett. 77, 4776 (1996).

[8] C. Baesens, S. Kim, and R. S. MacKay, Physica D (to be published); J. L. Marín, S. Aubry, and L. M. Floría, ibid. (to be published); L. Proville and S. Aubry ibid. (to be published).
[9] D. E. Pelinovsky, V. V. Afanasjev, and Yu. S. Kivshar, Phys. Rev. E 53, 1940 (1996); D. E. Pelinovsky, Yu. S. Kivshar, and V. V. Afanasjev, Physica D (to be published).

[10] We remark that since $H^{+}$and $H^{-}$are time independent, the approach used here is equivalent to the standard Floquet analysis that was used, e.g., in $[7,8,11]$. Namely, if $\mu$ is an eigenvalue of $\mathcal{M}$, then $\lambda=e^{\mu T}$, where $T=2 \pi / \Lambda$ is an eigenvalue of the Floquet matrix for the same eigenvector.

[11] S. Aubry, Physica D 103, 201 (1997).

[12] M. G. Vakhitov and A. A. Kolokolov, Izv. Vyssh. Uchebn. Zaved. Radiofiz. 16, 1020 (1973) [Sov. Radiophys. 16, 783 (1973)]; M. I. Weinstein, Comments Pure Appl. Math. 39, 51 (1986).

[13] Yu. B. Gaididei et al. (unpublished).

[14] We remark that a state close to the instability regime in general has a larger value of the Hamiltonian than its bistable counterpart. Thus, when $\left|\alpha_{\text {th }}\right|$ is small the switching can be viewed as a transition from a metastable state to a ground state. 